\title{
FERMAT'S PRINCIPLE FOR SEISMIC RAYS IN ELASTIC MEDIA
}

\author{
ANDREJ BÓNA AND MICHAEL A. SLAWINSKI
}

\begin{abstract}
We prove that rays in linearly elastic anisotropic nonuniform media obey Fermat's principle of stationary traveltime. First, we formulate the concept of rays, which emerges from the Hamilton equations. Then, we show that these rays are solutions of the variational problem stated by Fermat's principle. This proof is valid for all rays except the ones associated with inflection points on the phase-slowness surface.
\end{abstract}

Key words: Fermat's principle, seismic ray theory, elasticity, anisotropy, nonuniformity

\section{INTRODUCTION}

Fermat's variational principle of stationary traveltime is often implicitly assumed to be valid in seismological studies that employ ray-theory methods. Many quantitative results pertaining to traveltimes and raypaths are obtained using this principle. However, Fermat's principle is not a fundamental postulate of the ray theory and, thus, we set out to prove it for linearly elastic anisotropic nonuniform media.

Rays are solutions of the Hamilton equations. In many cases, rays can also be obtained by following Fermat's principle of stationary traveltime. In this paper, we use the rigorous notion of a ray, emerging from the Hamilton equations, to formulate and prove the statement of Fermat's principle.

Many researchers have discussed ray theory extensively. Notable works include Achenbach et al. (1982), Kravtsov and Orlov (1990), and Červený (2001). Our work is motivated by the works of Maurycy Pius Rudzki (Rudzki, 1913), Marcelo Epstein and Jędrzej Śniatycki (Epstein and Śniatycki, 1992) that deal with Fermat's variational principle. 
The paper of Rudzki (1913) emphasizes Fermat's principle as a variational problem in anisotropic nonuniform media, without actually proving this principle. Epstein and Śniatycki (1992) prove this principle by using a Hamiltonian that differs from the one used in our work and, hence, the resulting proof is different. The Hamiltonian in our work is a homogeneous function. This homogeneity significantly simplifies the computations involving the Hamiltonian.

We are also familiar with a proof by Babich (1961) in a paper that deals with the intensity of wavefronts in anisotropic nonuniform media. This paper, which relies on differential geometry, is written for an audience with substantial mathematical knowledge and requires the reader to identify the proof in the paper. Our approach, which shows the details of the proof of Fermat's principle in anisotropic nonuniform media, requires only familiarity with differential equations.

We would like to note that after the submission of our paper, a preprint of a new paper dealing with Fermat's principle was brought to our attention (Červený, 2002). In his paper, Červený (2002) invokes the concept of the Finsler metric, which defines the geometry of the calculus of variations in the context of ray theory (e.g., Antonelli et al., 2002), to prove Fermat's principle in anisotropic nonuniform media. The formulation of Červený (2002), similarly to the one of Babich (1961), is directed at readers familiar with differential geometry. Again, our approach relies on mathematical knowledge more familiar to a general audience of geophysicists.

\section{Statement of Fermat's PRinciple}

In 1657 , in a letter to Cureau de la Chambre ${ }^{1}$, Fermat formulated his variational principle for the propagation of light. He stated that light travels along a curve that renders the traveltime minimum. In modern notation, a generic form of this principle can be restated by the following theorem.

\footnotetext{
${ }^{1}$ Oeuvres de Fermat (Paris, 1891) Vol. II, p. 354.
} 
Theorem 1. Rays are the solutions of the variational problem

$$
\delta \int_{A}^{B} \frac{\mathrm{d} s}{V(x, n)}=0,
$$

where $\mathrm{d} s$ is an arclength element and $V(x, n)$ is the ray velocity in direction $n=$ $\mathrm{d} x / \mathrm{d} s$ at point $x . A$ and $B$ are the fixed endpoints of this variational problem.

Fermat's principle of stationary traveltime is often assumed to be valid for the propagation of seismic signals. However, the notions of a ray and the velocity, $V$, are not obvious entities. To obtain the meanings of these entities and prove Theorem 1, we formulate the concepts of ray and ray velocity ${ }^{2}$ in the context of linearly elastic anisotropic nonuniform media.

\section{SEISMIC RAYS}

3.1. Cauchy's equations of motion in elastic solids. Rays result from solving - by the method of characteristics - the eikonal equation, which is associated with the elastodynamic equations that correspond to a linearly elastic solid. The equations of the characteristics of the eikonal equation are the Hamilton equations, whose solutions give rays.

The elastodynamic equations are rooted in Cauchy's equations of motion, namely, ${ }^{3}$

$$
\rho(x) \frac{\mathrm{d}^{2} u^{i}}{\mathrm{~d} t^{2}}=\frac{\partial \sigma^{i j}}{\partial x^{j}}
$$

where $\rho(x)$ is the mass density at point $x, u$ is a vector describing the displacement of the medium, and $\sigma^{i j}$ is the stress tensor. As can be observed by considering the left-hand side of equation (3.1), Cauchy's equations of motion are a statement of Newton's second law.

\footnotetext{
${ }^{2}$ This entity is also commonly referred to as group velocity.

${ }^{3}$ In this paper we use the Einstein summation convention.
} 
To formulate the elastodynamic equations in the context of linear elasticity, we let the stress tensor be given by

$$
\sigma^{i j}=c^{i j k l}(x) \varepsilon_{k l},
$$

where $c^{i j k l}(x)$ are the elasticity parameters at point $x$, and

$$
\varepsilon_{k l}=\left(\partial u_{k} / \partial x^{l}+\partial u_{l} / \partial x^{k}\right) / 2
$$

is the infinitesimal strain tensor. Equations (3.2) are the constitutive equations of a linearly elastic solid.

Inserting the stress-tensor expression given in equations (3.2) into Cauchy's equations of motion, we obtain the desired elastodynamic equations. To solve these equations, we assume the trial solution to be of the form

$$
u(x, t)=A(x) f(\psi(x)-t),
$$

where $\psi$ is referred to as the eikonal function, whose level sets correspond to wavefronts, and where $A$ denotes the amplitude. Inserting trial solution (3.3) into the elastodynamic equations, we obtain

$$
\left\{\begin{array}{l}
\frac{\partial c^{i j k l}(x)}{\partial x^{j}} \frac{\partial A_{k}}{\partial x^{l}}+c^{i j k l}(x) \frac{\partial^{2} A_{k}}{\partial x^{j} \partial x^{l}}=0 \\
\frac{\partial}{\partial x^{j}}\left[c^{i j k l}(x) A_{l} \frac{\partial \psi}{\partial x^{k}}\right]+c^{i j k l}(x) \frac{\partial A_{k}}{\partial x^{l}} \frac{\partial \psi}{\partial x^{j}}=0 \\
c^{i j k l}(x) A_{k} \frac{\partial \psi}{\partial x^{j}} \frac{\partial \psi}{\partial x^{l}}-\rho(x) \delta^{i k} A_{k}=0
\end{array} .\right.
$$

Since the concepts of rays and ray velocity do not require us to consider amplitude, we turn our attention to the last equation of system (3.4) from which the amplitude can be factored out.

3.2. Eikonal equation. The last equation of system (3.4) possesses a nontrivial solution if and only if

$$
\operatorname{det}\left[c^{i j k l}(x) \frac{\partial \psi}{\partial x^{j}} \frac{\partial \psi}{\partial x^{l}}-\rho(x) \delta^{i k}\right]=0 \text {. }
$$


Considering a three-dimensional medium and denoting

$$
p_{j}=\frac{\partial \psi}{\partial x^{j}}
$$

we can write determinantal equation (3.5) as a third-degree polynomial in $p^{2}$, namely,

$$
\left(p^{2}\right)^{3} \operatorname{det}\left[c^{i j k l}(x) \frac{p_{j}}{|p|} \frac{p_{l}}{|p|}-\rho(x) \frac{1}{p^{2}} \delta^{i k}\right]=0 .
$$

As defined in expression (3.6), $p=\nabla \psi$ is a vector normal to the wavefront, and hence, in view of the trial solution, shown in expression (3.3), $p^{2}=p_{i} p^{i}$ is the squared magnitude of the slowness with which the wavefront propagates. Consequently, $v^{2}=1 / p^{2}$ is the squared magnitude of the velocity with which the wavefront propagates. Since wavefronts are loci of constant phase, this velocity is referred to as phase velocity.

Polynomial (??) can be written as

$$
\left[p^{2}-\frac{1}{v_{1}^{2}\left(x, \frac{p}{|p|}\right)}\right]\left[p^{2}-\frac{1}{v_{2}^{2}\left(x, \frac{p}{|p|}\right)}\right]\left[p^{2}-\frac{1}{v_{3}^{2}\left(x, \frac{p}{|p|}\right)}\right]=0 .
$$

Herein, the quantities $1 / v_{i}^{2}(x, p /|p|)$ are the three roots of polynomial (??), and, in general, correspond to the three wave types that exist in an anisotropic medium. Due to the positiveness of strain energy, the three roots are real and positive. For anisotropic solids, the three roots are distinct except at the discrete singular points, which commonly occur for the two slower waves. For isotropic solids, we obtain only two distinct roots. Since the dependence of $v$ on $p$ is given only in terms of $p /|p|$, the phase-velocity function, $v(x, p /|p|)$, is homogeneous ${ }^{4}$ of degree zero in $p$.

\footnotetext{
${ }^{4}$ We say that function $f$ is homogeneous of degree $r$ in $\xi$, if $f(c \xi, \zeta)=c^{r} f(\xi, \zeta)$, where $c$ denotes a constant. Similarly, function $f$ is absolute-value homogeneous of degree $r$ in $\xi$, if $f(c \xi, \zeta)=$ $|c|^{r} f(\xi, \zeta)$.
} 
Each factor of expression (3.8) corresponds to the eikonal equation for a given wave type, which can be written as

$$
p^{2}=\frac{1}{v^{2}\left(x, \frac{p}{|p|}\right)} .
$$

The eikonal equation is the key equation of ray theory. According to this equation, the squared magnitude of the slowness with which the wavefront propagates is a function of properties of the medium, which depend on position and direction.

Phase-velocity function, $v(x, p /|p|)$, is smooth and, hence, differentiable with respect to both position and direction. The smoothness with respect to position $x$ results from our assumption of a medium that is specified by functions $c^{i j k l}(x)$ and $\rho(x)$ that are smooth. The smoothness with respect to direction $p /|p|$ is a consequence of the smoothness of the multiplication and addition operations.

\subsection{Hamilton ray equations.}

3.3.1. Method of characteristics. In the context of ray theory, we solve equation (3.9) using the method of characteristics (e.g., Courant and Hilbert, 1989). To do so, we define function $H$, called the Hamiltonian, as

$$
H(x, p)=\frac{1}{2} p^{2} v^{2}\left(x, \frac{p}{|p|}\right)
$$

The factor of $1 / 2$ in expression (3.10) guarantees a parametrization of the characteristics of eikonal equation (3.9) by time.

The equations of the characteristics of eikonal equation (3.9) are the Hamilton equations, given by

$$
\left\{\begin{array}{l}
\dot{x}^{i}=\frac{\mathrm{d} x^{i}}{\mathrm{~d} t}=\frac{\partial H}{\partial p_{i}} \\
\dot{p}_{j}=\frac{\mathrm{d} p_{j}}{\mathrm{~d} t}=-\frac{\partial H}{\partial x^{j}}
\end{array} .\right.
$$

At this point, we can state the definition of a ray that underlies the formulation of this paper. 
Definition 2. Rays correspond to the solutions of the Hamilton equations, given by system (3.11). Rays are given by the function $x=x(t)$.

Remark 3. In mathematical literature, rays $x(t)$ are often referred to as base characteristics while $(x(t), p(t))$ are referred to as characteristics (e.g., Bleistein, 1984).

In view of Definition 2, the Hamilton equations that are shown in system (3.11) are often referred to as Hamilton ray equations. Definition 2 also allows us to state the concept of ray velocity $V$, which is given by $|\dot{x}(t)|$.

3.3.2. Properties of the Hamiltonian. By examining expression (3.10), we note that, in view of the phase velocity being homogeneous of degree zero in $p, H$ is homogeneous of degree two in $p$.

Also, since $H$ does not explicitly depend on time, its value is conserved along the ray. This can be stated by the following lemma.

Lemma 4. The Hamiltonian $H(x, p)$, given by expression (3.10), is conserved along the ray.

Proof. Using system (3.11) and the fact that $H$ does not explicitly depend on time, we can write

$$
\frac{\mathrm{d} H}{\mathrm{~d} t}=\frac{\partial H}{\partial x} \dot{x}+\frac{\partial H}{\partial p} \dot{p}+\frac{\partial H}{\partial t}=-\dot{p} \dot{x}+\dot{x} \dot{p}=0
$$

The value of the Hamiltonian, which is conserved along the ray, is equal to $1 / 2$. This results from the fact that the eikonal equation, which is shown in equation (3.9), must be satisfied along its characteristics. Hence, in view of this equation, which states that $p^{2} v^{2}=1$, and expression (3.10), we require that

$$
H(x, p)=\frac{1}{2},
$$

along a ray. 


\section{VARIATIONAL FORMULATION}

4.1. Euler equations ${ }^{5}$. Fermat's principle is a variational formulation. To show that this principle is valid for seismic rays, we use the Legendre transformation to restate the Hamilton ray equations as the Euler equations, which state the stationarity condition of a variational problem.

In view of the Legendre transformation, we can define the Lagrangian as

$$
L(x, \dot{x})=p_{i}(x, \dot{x}) \dot{x}^{i}-H(x, p(x, \dot{x})),
$$

where

$$
\dot{x}^{i}=\frac{\partial H}{\partial p_{i}},
$$

is the inverse Legendre transformation. Having defined $L$, we can state the raytracing problem in the context of the calculus of variations, namely,

$$
\delta \int_{A}^{B} L \mathrm{~d} t=0 .
$$

Hence, the rays satisfy the Euler equations

$$
\frac{\partial L}{\partial x^{i}}-\frac{\mathrm{d}}{\mathrm{d} t}\left(\frac{\partial L}{\partial \dot{x}^{i}}\right)=0
$$

In our case, the Lagrangian, shown in expression (4.1), is conserved along each ray and its value is $1 / 2$. Also, this Lagrangian is homogeneous of degree two in $\dot{x}$. These properties are shown in the following steps.

Lemma 5. If $H(x, p)$ is homogeneous of degree two in $p$, then

$$
L(x, \dot{x}(x, p))=H(x, p)
$$

where, by the inverse Legendre transformation, stated in expression (4.2), $\dot{x}^{i}=$ $\partial H / \partial p_{i}$

\footnotetext{
${ }^{5}$ These equations are also commonly referred to as the Euler-Lagrange equations.
} 
Proof. Consider the Lagrangian

$$
L(x, \dot{x}(x, p))=p_{i} \dot{x}^{i}-H .
$$

In view of the inverse Legendre transformation, we can write

$$
L(x(t), \dot{x}(x(t), p(t)))=p_{i} \frac{\partial H}{\partial p_{i}}-H .
$$

If $H$ is homogeneous of degree two in $p$, by Euler's homogeneous-function theorem, we obtain

$$
L(x(t), \dot{x}(x(t), p(t)))=2 H-H=H,
$$

which is the required expression.

In view of the conserved value of the Hamiltonian, $H$, as shown in Lemma 4 and, following expression (3.12), we obtain the following corollary of Lemma 5.

Corollary 6. Along each ray, the Lagrangian, $L$, is equal to $1 / 2$.

In our case, in view of the Hamiltonian being homogeneous of degree two in $p$, the analogous property of the Lagrangian is shown in the following lemma.

Lemma 7. If the Hamiltonian, $H(x, p)$, is homogeneous of degree two in $p$, then the Lagrangian, $L(x, \dot{x})$, is homogeneous of degree two in $\dot{x}$.

Proof. By Lemma $5, H(x, p)=L(x, \dot{x}(x, p))$, where $\dot{x}$ and $p$ are related by the inverse Legendre transformation $\dot{x}^{i}=\partial H / \partial p_{i}$. Let $p^{\prime}=a p$, where $a$ is a constant. The corresponding Hamilton equations are

$$
\left(\dot{x}^{\prime}\right)^{i}=\frac{\partial H\left(x, p^{\prime}\right)}{\partial p_{i}^{\prime}}=\frac{\partial H(x, a p)}{\partial\left(a p_{i}\right)} .
$$

By the homogeneity of $H$ and the property of the differential operator, we can write

$$
\left(\dot{x}^{\prime}\right)^{i}=\frac{\partial H(x, a p)}{\partial\left(a p_{i}\right)}=\frac{a^{2} \frac{\partial H(x, p)}{\partial p_{i}}}{\frac{\partial\left(a p_{i}\right)}{\partial p_{i}}}=\frac{a^{2} \frac{\partial H(x, p)}{\partial p_{i}}}{a} ;
$$


hence,

$$
\left(\dot{x}^{\prime}\right)^{i}=a \frac{\partial H(x, p)}{\partial p_{i}},
$$

which, in view of the Hamilton equations, given by system (3.11), can be stated as

$$
\dot{x}^{\prime}=a \dot{x} .
$$

Consequently, we can write

$$
L(x, a \dot{x})=L\left(x, \dot{x}^{\prime}\right)
$$

which, by Lemma 5, yields

$$
L(x, a \dot{x})=L\left(x, \dot{x}^{\prime}\right)=H\left(x, p^{\prime}\right)=H(x, a p)=a^{2} H(x, p)=a^{2} L(x, \dot{x}),
$$

where the expression in the middle results from the homogeneity of $H$. This means that the Lagrangian $L(x, \dot{x})$ is homogeneous of degree two in $\dot{x}$.

The solutions of system (4.4) have a fixed parametrization by the traveltime. In other words, variational problem (4.3) depends on parametrization. For the Euler equations to be independent of parametrization, the corresponding Lagrangian must be absolute-value homogeneous of degree one in $\dot{x}$. Parametrization independence is necessary to state Fermat's principle since its generic form, as shown in expression (2.1), is parametrization independent.

Let us consider function $F$ given by

$$
F=\sqrt{2 L}
$$

Note that $F$ is absolute-value homogeneous of degree one in $\dot{x}$. Under certain conditions, which are satisfied in our case, the solutions of the Euler equations given by system (4.4) are also the solutions of the Euler equations given by

$$
\frac{\partial F}{\partial x^{i}}-\frac{\mathrm{d}}{\mathrm{d} t}\left(\frac{\partial F}{\partial \dot{x}^{i}}\right)=0 .
$$

This is stated by the following lemma. 
Lemma 8. A solution of equations (4.4) that satisfies the initial condition given in Corollary 6, where $L$ is given by expression (4.1) is also a solution of equations (4.6), where $F=\sqrt{2 L}$.

Proof. Inserting $L=F^{2} / 2$ into equations (4.4), we obtain

$$
\begin{aligned}
\frac{\partial}{\partial x^{i}}\left(\frac{F^{2}}{2}\right)-\frac{\mathrm{d}}{\mathrm{d} t}\left[\frac{\partial}{\partial \dot{x}^{i}}\left(\frac{F^{2}}{2}\right)\right] & =F \frac{\partial F}{\partial x^{i}}-\frac{\mathrm{d}}{\mathrm{d} t}\left[F \frac{\partial F}{\partial \dot{x}^{i}}\right] \\
& =F\left[\frac{\partial F}{\partial x^{i}}-\frac{\mathrm{d}}{\mathrm{d} t}\left(\frac{\partial F}{\partial \dot{x}^{i}}\right)\right]-\frac{\mathrm{d} F}{\mathrm{~d} t} \frac{\partial F}{\partial \dot{x}^{i}} \\
& =0 .
\end{aligned}
$$

Since $L=1 / 2$ along a ray, as shown in Corollary 6 , then $F=1$ and, hence, $\mathrm{d} F / \mathrm{d} t=0$ along the solutions of equations (4.4). Thus, equations (4.4) become equations (4.6), as required.

If we can show that

$$
F=\frac{|\dot{x}|}{V\left(x, \frac{\dot{x}}{|\dot{x}|}\right)},
$$

where $|\dot{x}|=\mathrm{d} s / \mathrm{d} t$ and $\dot{x} /|\dot{x}|=n$, then we prove Theorem 1 , since the right-hand side of equation (4.7) is the integrand of equation (2.1).

4.2. Ray velocity. In order to show that the right-hand side of equation (4.7) is the integrand of equation (2.1), we must formulate ray velocity in a variational context. Since, as shown in Lemma 7, the Lagrangian is homogenous of degree two in $\dot{x}$, we can write

$$
L(x, \dot{x})=L(x,|\dot{x}| n)=|\dot{x}|^{2} L(x, n),
$$

where $n=\dot{x} /|\dot{x}|$. Since, as stated in Corollary 6 , the value of the Lagrangian along a ray is $1 / 2$, we can write

$$
\frac{1}{2}=|\dot{x}|^{2} L(x, n)
$$


As this expression is valid along any ray, the ray velocity $V$, given by $|\dot{x}|$, can be expressed as

$$
V(x, n)=|\dot{x}|=\frac{1}{\sqrt{2 L(x, n)}} .
$$

Now, we are ready to complete our proof of Theorem 1.

\section{Proof of Theorem 1}

Proof. By Lemma 8, rays are the solutions of the Euler equations stated in system (4.6). Consequently, the rays are the solutions of the variational problem

$$
\delta \int_{A}^{B} F \mathrm{~d} t=0
$$

In view of expression (4.5), we can restate this variational problem as

$$
\delta \int_{A}^{B} F(x, \dot{x}) \mathrm{d} t=\delta \int_{A}^{B} \sqrt{2 L(x, \dot{x})} \mathrm{d} t=0 .
$$

Since, as stated in Lemma $7, L$ is homogeneous of degree two in $\dot{x}$, we can write

$$
\delta \int_{A}^{B} \sqrt{2|\dot{x}|^{2} L(x, n)} \mathrm{d} t=\delta \int_{A}^{B}|\dot{x}| \sqrt{2 L(x, n)} \mathrm{d} t=0 .
$$

In view of expressions (4.8) and recalling that $|\dot{x}| \mathrm{d} t=\mathrm{d} s$, we conclude that

$$
\delta \int_{A}^{B} F(x, \dot{x}) \mathrm{d} t=\delta \int_{A}^{B} \frac{\mathrm{d} s}{V(x, n)}=0 .
$$

Hence, the solutions of the Hamilton ray equations, which, by Definition 2, correspond to rays, are the solutions of variational problem (2.1).

\section{Conclusions}

We have proven that, in general, seismic rays in linearly elastic anisotropic nonuniform media obey Fermat's principle of stationary traveltime. Firstly, we formulate the concept of seismic rays, which emerges from the Hamilton equations. 
Secondly, we show that these rays satisfy the generic form of Fermat's variational principle.

Our proof relies on the Legendre transformation. We can use the Legendre transformation only if the Hamiltonian, $H$, is regular, namely,

$$
\operatorname{det}\left[\frac{\partial^{2} H}{\partial p_{i} \partial p_{j}}\right] \neq 0
$$

As stated by Hanyga (2001), the requirement of regularity imposes certain limitations on our ability to prove Theorem 1; namely, we are unable to use the Legendre transformation at the inflection points. Note that this limitation applies to all the proofs of Fermat's principle that are known to us. However, as shown by Musgrave (1970), for an elastic medium defined by constitutive equations (3.2), the innermost phase-slowness surface is always convex and, hence, the Hamiltonian associated with the fastest wave is always regular. In other words, Fermat's principle is always valid for the fastest wave. For the other two wave types, we cannot use our approach at the points where the Hamiltonian is irregular.

This does not mean that Fermat's principle does not hold in general; however, the proof of Theorem 1 in the context of a phase-velocity function giving an irregular Hamiltonian remains an open problem.

\section{ACKNOWLEDGEMENTS}

We wish to thank Nelu Bucataru and Jędrzej Śniatycki for numerous fruitful discussions on this topic. We are also grateful to Cathy Beveridge for her editorial work.

This study has been done with the support of The Geomechanics Project. Hence, the authors wish to acknowledge the support and encouragement of EnCana Energy and Talisman Energy.

We would also like to thank the two anonymous reviewers and Ivan Pšenčík (editor) for their excellent comments, which enhanced the quality of this paper. 


\section{REFERENCES}

Achenbach,: J.D., Gausten, A.K., and McMaken, H., 1982. Ray methods for waves in elastic solids: with applications to scattering by cracks. Pitman Publishing Inc., London.

Antonelli,: P.L., Bóna, A., and Slawinski, M.A., 2002. Seismic Rays as Finsler Geodesics. Nonlinear Analysis (in press).

Babich,: V.M., 1961. Ray method of calculating the intensity of wavefronts in the case of a heterogeneous, anisotropic, elastic medium. In: G.I. Petrashen (ed.), Problems of the dynamic theory of propagation of seismic waves (in Russian), 5, 36 - 46. Leningrad University Press. (English translation: Geophys. J. Int., 1994, 118, $379-383$.

Bleistein,: N., 1984. Mathematical methods for wave phenomena. Academic Press, San Diego, CA.

Červený,: V., 2001. Seismic ray theory. Cambridge University Press, Cambridge.

Červený,: V., 2002. Fermat's variational principle for anisotropic inhomogeneous media. Studia geophysica and geodætica, 46, $567-588$.

Courant,: R., and Hilbert, D., 1989. Methods of mathematical physics, Vol. II. John Wiley \& Sons, NY.

Epstein,: M., Śniatycki, J., 1992. Fermat's principle in elastodynamics. Journal of Elasticity, 27, $45-56$.

Hanyga,: A., 2001. Fermat's principle in anisotropic elastic media. In: J.A. Hood (ed.), Advances in anisotropy: Selected theory, modeling, and case studies. Society of Exploration Geophysicists, $271-288$.

Kravtsov,: Y.A., and Orlov, Y.I., 1990. Geometrical optics of inhomogeneous media. Springer-Verlag, Heidelberg.

Musgrave,: M.J.P., 1970. Crystal acoustics: Introduction to the study of elastic waves and vibrations in crystals. Holden-Day, San Francisco. 
Rudzki,: M.P., 1913. Essai d'application du principe de Fermat aux milieux anisotropes: Mémoir. (English translation: On application of Fermat's principle to anisotropic media. In: L. Ikelle and A. Gangi (eds.), Anisotropy 2000: Fractures, converted waves, and case studies, 13 - 20. Society of Exploration Geophysicists, (Special Issue).

Department of Earth Sciences, Memorial University of Newfoundland, Canada

E-mail address: andrej@lagrange.esd.mun.ca

Department of Earth Sciences, Memorial University of Newfoundland, Canada

E-mail address: mslawins@mun.ca 